\title{
Taller de creatividad para todo el centro escolar
}

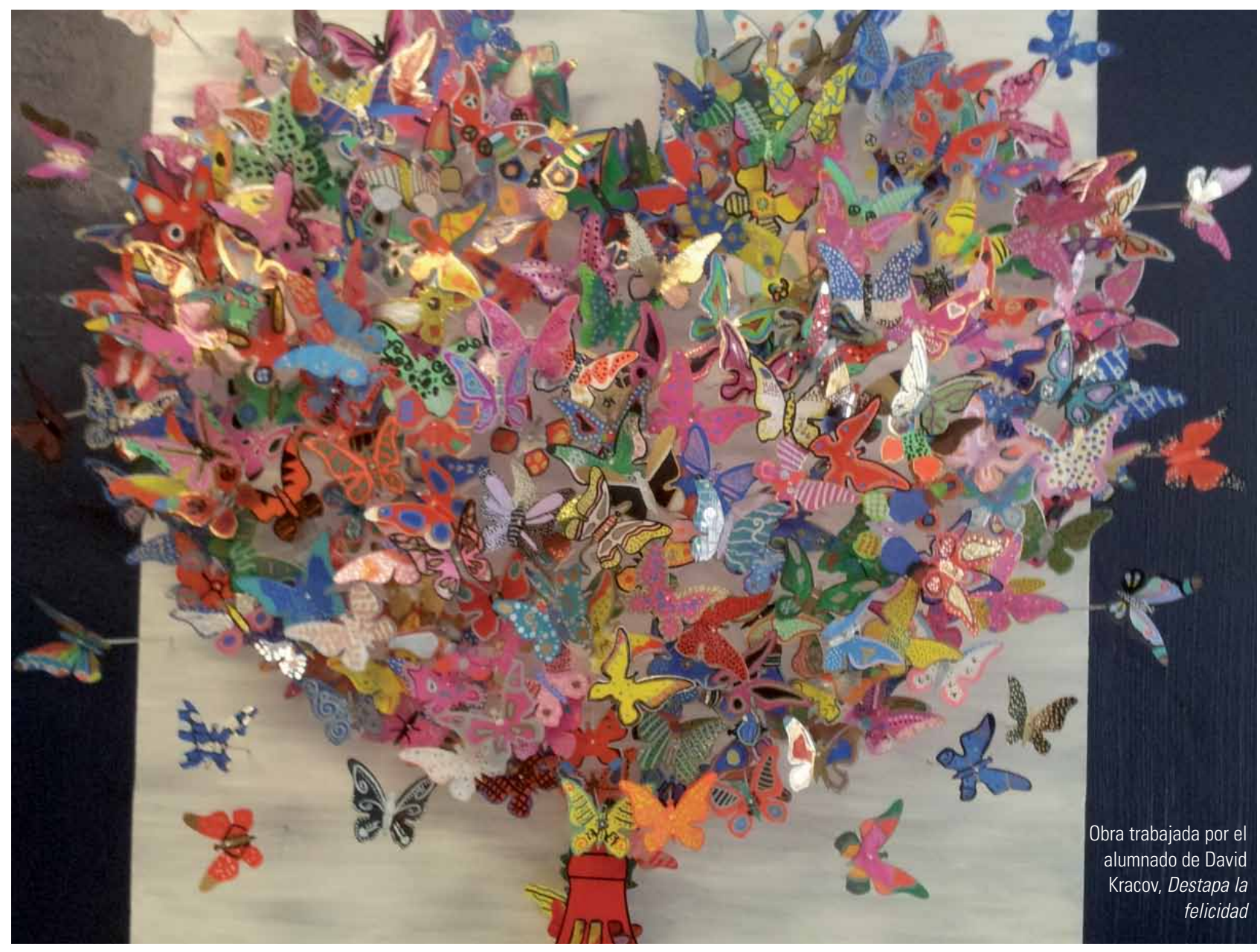

El interés del proyecto estriba no sólo en su carácter innovador, en la medida que trasciende el propio contenido y por el que queda afectada la metodología, sino también en criterios específicos como la mayor participación de los estudiantes, el establecimiento de espacios y

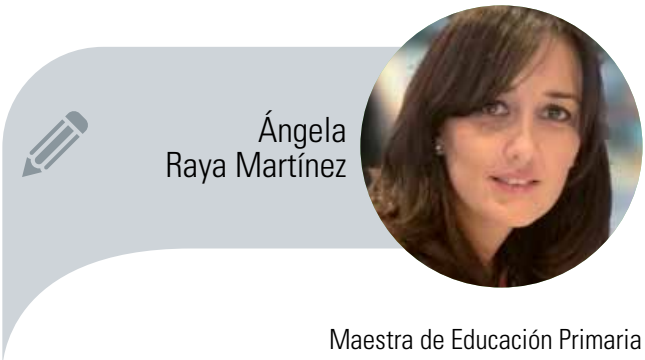

CEIP Jacinto Benavente de Alcantarilla (Murcia) mangel.raya@murciaeduca.es tiempos comunes entre el alumnado de distintas edades y sobre todo intenciones educativas compartidas que permiten el aprendizaje colaborativo. 


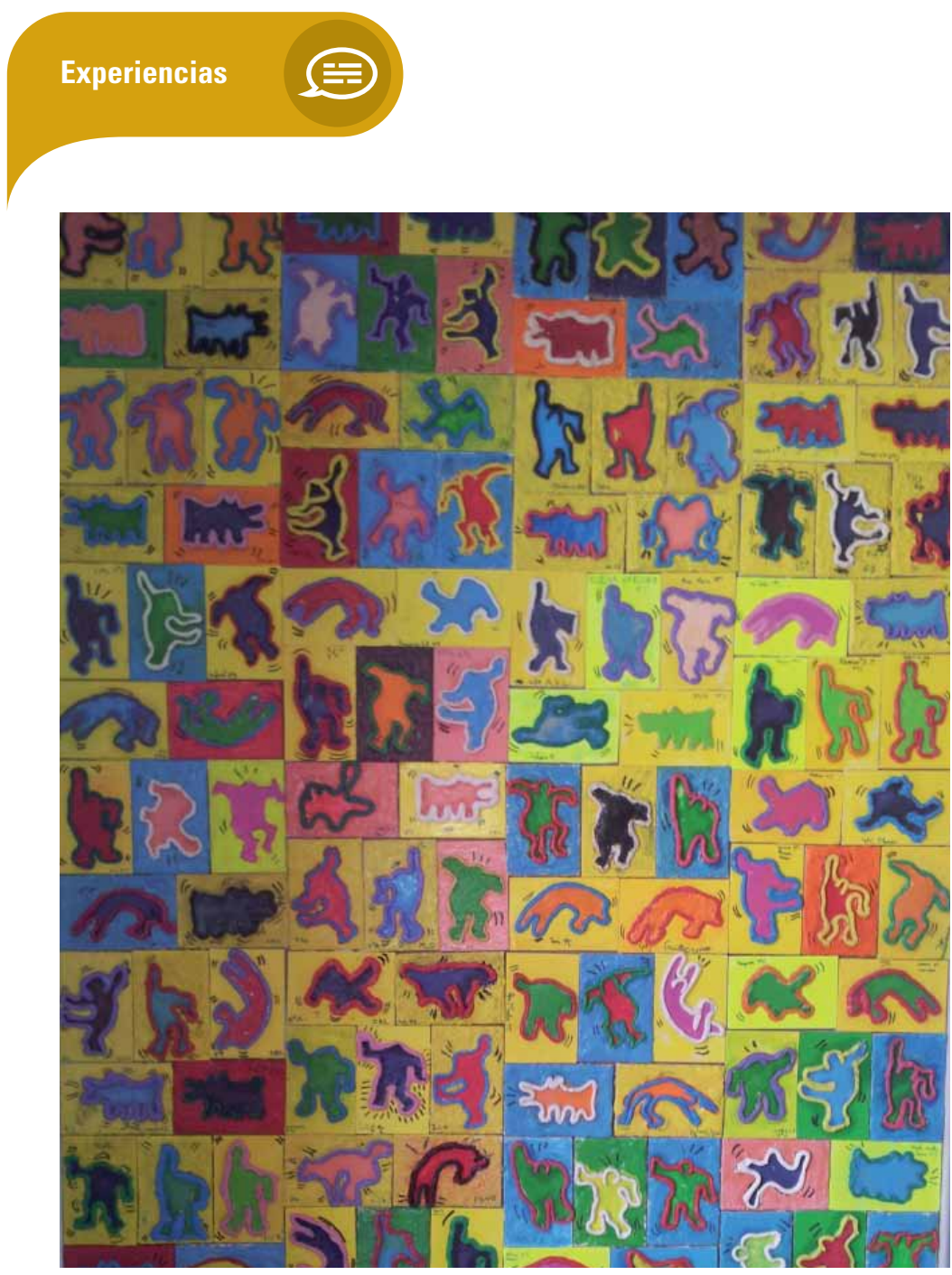

Como todo centro educativo, este curso académico nos hemos visto envueltos en un proceso de cambio surgido a raíz de la implantación de la nueva normativa legislativa, tanto a nivel nacional como regional.

Entre la farragosa legislación y el conglomerado de cambios asociados a ella, el profesorado del CEIP Jacinto Benavente de Alcantarilla (Murcia) encontró un elemento catalizador sobre el que podría discurrir el cambio global que lleva asociada toda nueva normativa educativa. El carácter facilitador del cambio y de la mejora que supone la creatividad y la innovación educativa nos permitió encontrar en ellas un punto de apoyo en el que intensificar nuestra dedicación durante este año. El proyecto que a continuación presentamos surge a raíz de la publicación de la Orden de 20 de noviembre de 2014 por la que se regula la organización y la evaluación en la Educación Primaria en nuestra región, la cual nos señala en su artículo 11 que entre los espacios del centro se podría configurar un aula específica de la creatividad que promueva la autonomía, el emprendimiento, el aprendizaje autónomo, la originalidad y el pensamiento crítico.

Sustentados en la normativa, y respetando la particularidad del contexto socioeducativo en el que desarrollamos nuestra labor docente, diseñamos un proyecto denominado "Taller de crea-

\section{La crealividad es la generación}

\author{
de una idea y la innovación es el \\ desarrollo, la explotación máxima
}

de la idea, aquí aparece la

innovación crealiva

tividad", en el que participa todo el alumnado del centro.

EI CEIP Jacinto Benavente de Alcantarilla (Murcia) es un centro de cuatro líneas, desde su etapa de infantil hasta la primaria, tiene un aula abierta con 6 alumnos, es un centro de integración que presenta 49 alumnos con problemáticas diversas de autismo, síndrome de Down, Asperger, motóricos, entre otros menos comunes y 32 alumnos de altas capacidades. Es, por tanto, evidente el volumen de alumnos y su diversidad.

El interés del proyecto estriba pues no sólo en su carácter innovador, en la medida que trasciende el propio contenido y por el que queda afectada la metodología, sino también en criterios específicos como la mayor participación de los estudiantes, el establecimiento de espacios y tiempos comunes entre alumnos y alumnas de distintas edades y sobre todo intenciones educativas compartidas que permiten el aprendizaje colaborativo.

Estos principios pedagógicos sobre los que se sustenta nuestra propuesta exigían que a la hora de plantear la actividad se establecieran elementos comunes que a todos les despertaran la capacidad de generar formas no habituales de hacer las cosas, de resolver problemas o de abordar las situaciones de manera diferente.

El objetivo general es generar una conciencia social, de manera que el alumnado se sienta parte del centro, que disfruten con la elaboración de proyectos comunes, respetando las aportaciones de los demás, los espacios y materiales que compartimos.

No podemos hablar de creatividad sin el concepto de innovación. La convergencia de ambos nos lleva a la innovación creativa. Y es que toda innovación requiere de la creatividad. Tengamos en cuenta que la creatividad es la generación de una idea y la innovación es el desarrollo, la explotación máxima de la idea, aquí es cuando realmente apa- 


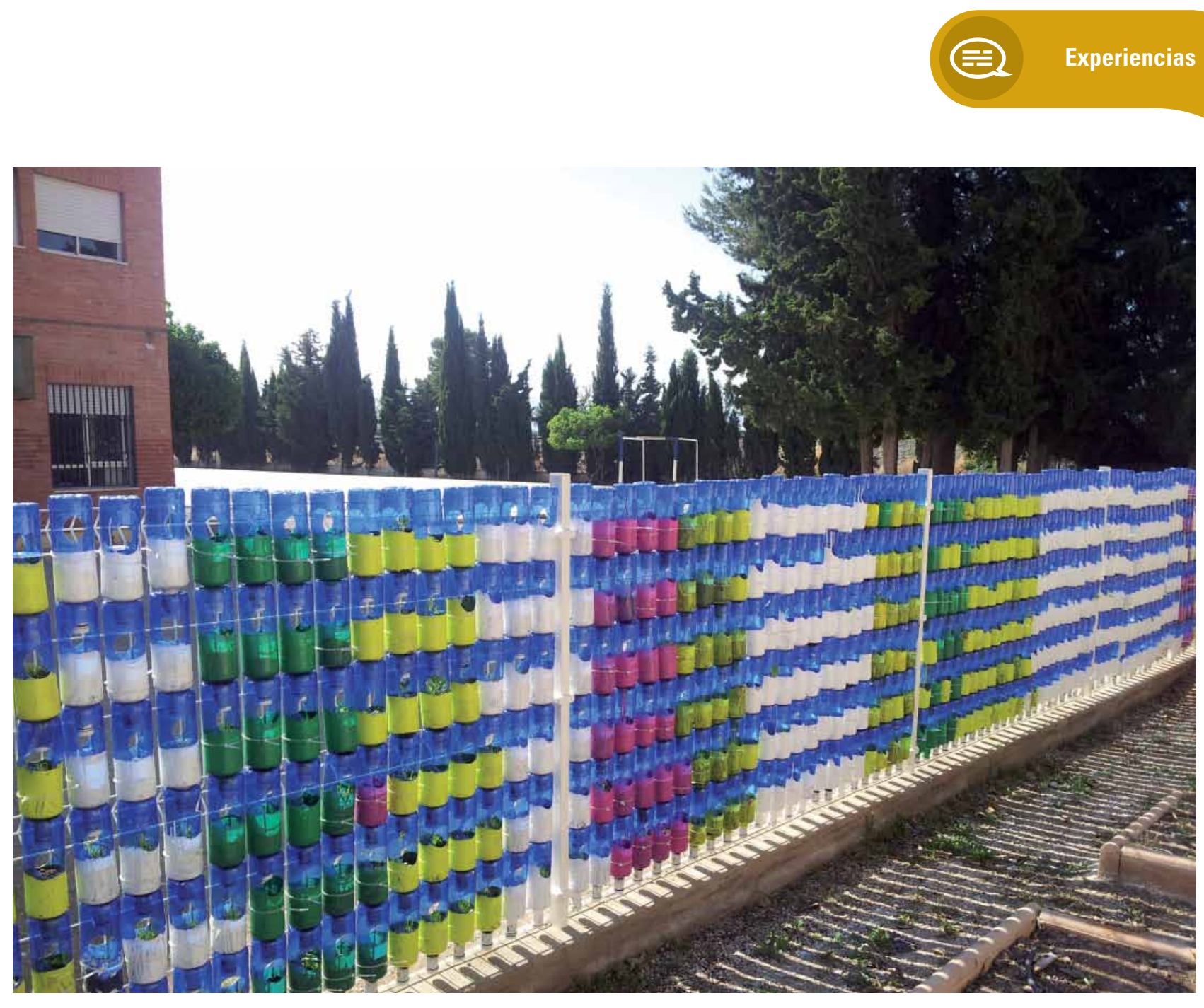

rece la innovación creativa. En definitiva, era lo que buscábamos con este proyecto, que el alumnado se entusiasmase con algo nuevo, que le permitiese crear, imaginar y desarrollar. Para que el alumnado lo percibiese como algo nuevo, debía sentir el deseo de volver al taller, de pensar en el trabajo que se estaba desarrollando en él y de trabajarlo en otros momentos fuera del aula. Para conseguir esto, había que innovar en la creatividad con la que se comunicaba y se desarrollaba en el trabajo.

La experiencia aún se está desarrollando, pero nos ha confirmado en nuestros planteamientos en la medida que los estudiantes han encontrado en este taller algo novedoso, ha creado un espacio en el que poder pensar de manera diferente, trabajar con diferentes reglas a las propias del aula y se han sentido partícipes de un proyecto global para todo el centro. Han sentido la magia de la creatividad.

Este proyecto no renuncia al aprendizaje por competencias, pero se caracteriza por ser un aprendizaje transversal, dinámico e integral, que se adapta al ritmo de trabajo de los alumnos. Es un proyecto interdisciplinar, por lo que las sesiones se llevan a cabo en diferentes áreas. Al ser un proyecto en común para todo el centro, se habilitó un espacio en el centro, lo que antes era el laboratorio, pasó a ser el "aula de creatividad" y como sala de exposición de los trabajos desarrollados se emplea todo el centro.

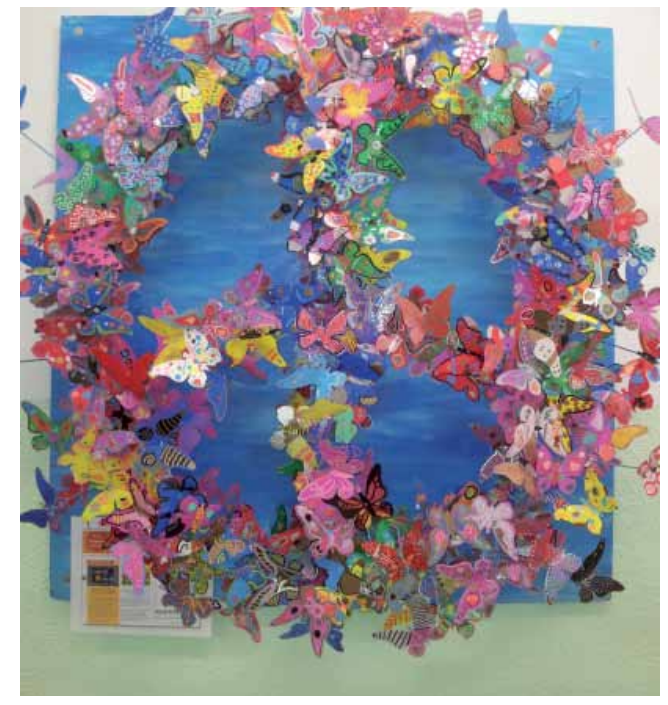

Huerto vertical escolar

El proyecto viene dividido en tres subproyectos:

7 Arte contemporáneo.

$\boldsymbol{7}$ Medio ambiente.

$\boldsymbol{\lambda}$ Teatro.

A través del proyecto de arte contemporáneo se ha pretendido entre otros objetivos disfrutar con la manipulación y exploración de diferentes técnicas, materiales, objetos e instrumentos para la creati- 

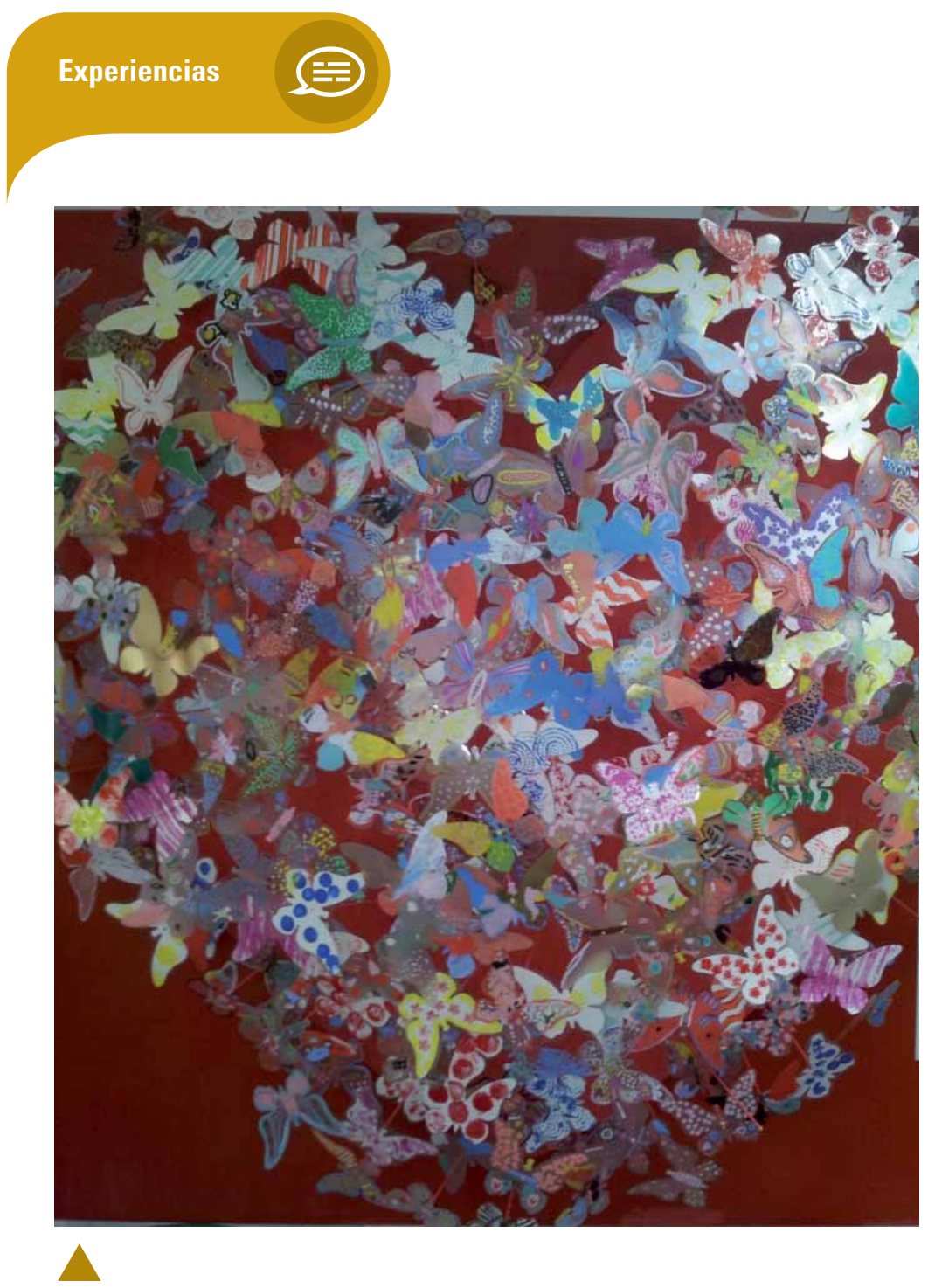

Corazón

vidad artística, explorando así las posibilidades expresivas y estéticas que ofrecen, desarrollar la autoconfianza y valoración de las producciones artísticas personales, respetando al mismo tiempo la de los demás, no olvidando que la obra que desarrollada por el compañero formaría parte de su propia obra. Se pretendía además despertar la inquietud y el afán por la realización de obras personales y desarrollar una actitud crítica ante las diferentes obras artísticas. Se ha trabajado en base a la obra de tres artistas contemporáneos dando a conocer al autor, su estilo, trabajo y la corriente artística a la que pertenecían. Estos autores han sido Wassily Kandinsky, Keith Haring y David Kracov.

El primer tramo de Primaria (un total de 300 alumnos) más los alumnos del aula abierta, trabajaron sobre la obra de Kandinsky titulada Círculos concéntricos. En una primera sesión, los alumnos de altas capacidades de este tramo desarrollaban una presentación del artista para darlo a conocer al resto de sus compañeros. Esta elaboración de información se trabajaba en las sesiones de profundización que se realizan dentro del proyecto de altas capacidades. En esta primera sesión se exponía la biografía del artista y se ubicaba dentro de la corriente artística y sus características a través de sus obras. En esta sesión daba comienzo la réplica que entre todo el alumnado se iba a hacer

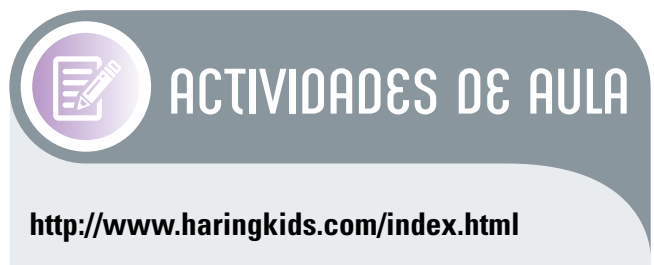

Web diseñada para que los niños puedan diseñar sus propios trabajos sobre el artista Keith Haring. Son múltiples las actividades con las que pueden trabajar y despertar su imaginación.

de su obra. Para toda la composición se necesitó de tres sesiones de una hora. Quedando una obra final de tres por dos metros, compuesto de 300 cuadriláteros de cartón pluma de diez por diez centímetros, en cuyo centro quedan los círculos concéntricos de plastilina de diferentes colores.

Para el segundo tramo, se realizó una réplica de la obra del contemporáneo Keith Haring. Sus dibujos eran más conocidos por el alumnado, ya que lo encuentran en diferentes objetos de la vida cotidiana: prendas deportivas, vajillas, murales, etc., pero hasta ahora no se habían planteado el uso de los mismos para diferentes situaciones y la capacidad que tenía Haring para transformar a la actualidad el uso de los jeroglíficos egipcios. El desarrollo de este taller fue análogo al del primer tramo, con tres sesiones, en la que parte de la primera se utilizó para conocer al artista a cargo de los alumnos que participan en el programa de altas capacidades. En esta ocasión se realizan dos réplicas de la obra de tres por dos metros, debido al peso de la misma. Está compuesta cada una de ellas por 150 piezas de cartón pluma de diez por quince centímetros en las que aparece una de las diferentes figuras en movimiento de Haring, realizadas con una masa hecha con marmolina y cola. La silueta y el movimiento propio de la figura lo realizan con plastilina.

Para el tercer proyecto de arte contemporáneo ampliamos la participación a los alumnos de educación infantil. Esto fue debido al entusiasmo que despertó la realización del proyecto anterior no sólo en el alumnado, sino también entre el profesorado y las familias. Además, nuestra propuesta estaba vinculada al día de la Paz, en la que también participa todo el centro. Para ello nos apoyamos en las obras de David Kracov, el cual trabaja con las mariposas y el concepto del proverbio chino "efecto mariposa". Para este proyecto utilizamos la misma metodología, pero trabajamos con diferentes materiales, ya que había que desarrollarlo con niños de la etapa de infantil. Fueron tres las obras que pudimos obtener de las más de 900 mariposas elaboradas por nuestros 
alumnos. Cada mariposa va clavada en un palo de madera y éste a su vez en la base que conforma la obra. La sorpresa llegó cuando enviamos la foto del resultado a la página oficial de Facebook de Kracov y obtuvimos respuesta del mismo, agradeciendo nuestro trabajo y el entusiamo que los niños habían depositado en él.

Una vez finalizado este proyecto pasamos al proyecto de medio ambiente, cuya finalidad es la de concienciar a los alumnos del uso sostenible del agua en el Planeta y en particular en la región de Murcia. Para ello presentamos la alternativa al huerto tradicional, ya presente en nuestro centro. Les planteamos incrementar el aprovechamiento del agua con respecto al huerto tradicional que ya funcionaba. Propusimos como alternativa la elaboración de un huerto vertical, en el que con un sistema de regadío en cadena podríamos obtener un ahorro de agua. Además de que cada alumno pudiera cuidar no sólo de su plantación; sino también de la de los que formaban parte de su cadena.

Para ello se ha fabricado entre todos un huerto vertical a través de botellas encadenadas, teniendo en cuenta las diferentes características que debía presentar cada botella para el desarrollo adecuado de la vegetación. Para todo este proceso se ha hecho uso de dos sesiones quincenales de una hora.

En la actualidad nos encontramos desarrollando el proyecto de teatro, con él se quiere promover la confianza en uno mismo, la expresión emocional, la comunicación, la imaginación, la creatividad y el trabajo en equipo. Un niño sin una inteligencia emocional desarrollada y adecuada, difícilmente será creativo.

Consideramos fundamental poner al alcance del alumnado todos los lenguajes que le sirvan para expresarse a nivel emocional: el lenguaje musical, el plástico, el verbal, el no verbal, el simbólico. Y en el aula de creatividad creemos encontrar un marco apropiado para el desarrollo de estos de manera creativa e innovadora.

Para finalizar, señalar que en este proyecto se trabajará las aportaciones del teatro a las emociones, a través de la elaboración de los diferentes elementos como la representación, trabajando su vocabulario, los diferentes personajes que pueden participar, la expresión corporal. Nos basaremos en el trabajo de grandes personajes del teatro de las emociones, Charlot, Charlie Rivel y Marcel Marceau. Y se elaborará un libro de las emociones, El emocionario •
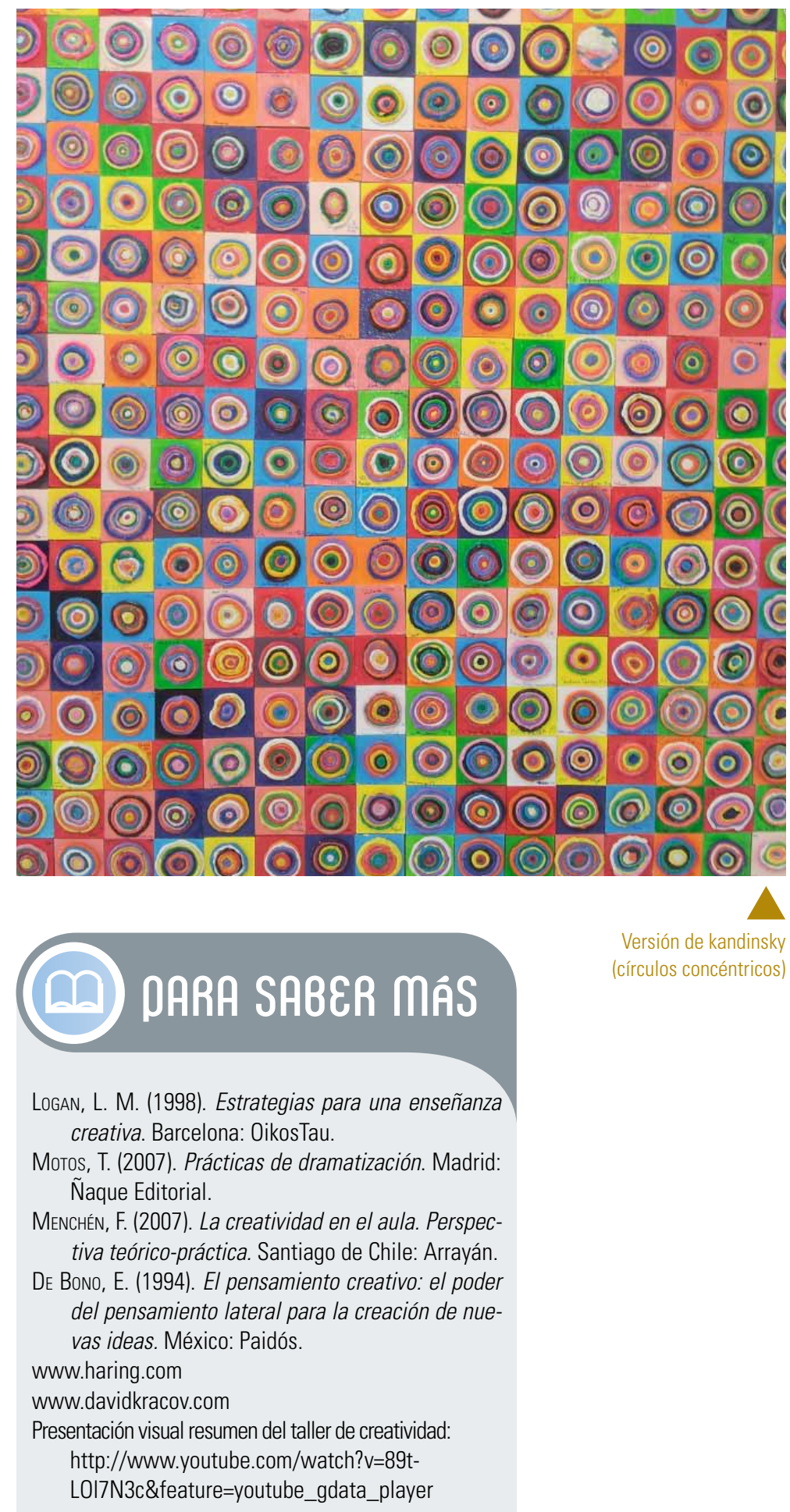

Versión de kandinsky (círculos concéntricos)

\section{Creatividad, innovación, aprendizaje, competencias, arte, teatro, medioambiente.}

Este artículo fue solicitado por PADRES y MAESTROS en diciembre de 2014, revisado y aceptado en marzo de 2015. 